\title{
Mesures de propriétés non linéaires thermiques de couches minces diélectriques
}

\author{
S. Monneret et F. Flory \\ Laboratoire d'Optique des Surfaces et des Couches Minces, École Nationale Supérieure \\ de Physique, Domaine Universitaire de Saint-Jérôme, 13397 Marseille cedex 20, France
}

\begin{abstract}
Résumé - Un appareillage basé sur le coupleur à prisme à deux faisceaux est utilisé pour mesurer les temps caractéristiques et l'amplitude d'instabilités photo-induites dans des matériaux diélectriques déposés en couches minces. Les résultats révèlent divers phénomènes d'origine thermique.
\end{abstract}

\section{INTRODUCTION}

Les matériaux diélectriques déposés en couches minces et utilisés pour la réalisation de filtres interférentiels performants présentent des pertes par diffusion et par absorption très faibles. Ils permettent d'ailleurs de réaliser des guides d'onde plans dont les pertes totales peuvent être inférieures à $1 \mathrm{~dB} / \mathrm{cm}$.

Malgré ces faibles pertes, l'indice de réfraction de ces matériaux peut être modifié sous l'effet de la lumière [1]. Ces modifications peuvent être mesurées dans les couches minces présentant des modes guidés. On bénéficie effectivement à la fois de résonances marquées des ondes optiques et de grandes longueurs d'interaction lumière-matière. Ainsi, à partir d'un faisceau de puissance moyenne modérée, on peut confiner une très forte intensité lumineuse dans l'épaisseur de la couche mince du matériau étudié.

\section{MONTAGE EXPÉRIMENTAL}

La méthode employée pour obtenir de telles résonances est une technique basée sur l'utilisation d'un coupleur à prisme à réflexion totale, développée depuis plusieurs années au laboratoire. Cette méthode repose sur l'observation de lignes de modes apparaissant dans le faisceau réfléchi par le système (figure 1), lorsque l'angle $\theta$ d'incidence moyenne du faisceau permet l'excitation d'un champ résonnant dans la couche mince. Cette méthode est souvent utilisée pour caractériser des couches minces (indice de réfraction, épaisseur, anisotropie,...)[2].

On peut aussi coupler un deuxième faisceau, de forte puissance, dans la couche mince. Ce faisceau (faisceau pompe) va pouvoir provoquer des instabilités réversibles dans le guide, qui vont perturber le profil d'intensité de la ligne de mode du faisceau initial (faisceau sonde). Ces modifications sont enregistrées directement par un photodétecteur de petites dimensions, placé sur ce profil. La modulation en créneaux du faisceau pompe, à basse fréquence (20$1000 \mathrm{~Hz}$ ), nous permet d'étudier à la fois les temps de montée et de descente des perturbations photo-induites par le faisceau pompe. L'analyse des phénomènes en fonction de la fréquence 
de modulation du faisceau pompe et de sa puissance nous donne alors des renseignements sur les mécanismes responsables des perturbations.

\section{RÉSULTATS}

Avec un champ sonde de petites dimensions devant le champ pompe, on peut se contenter d'un modèle linéaire pour interpréter les mesures. Ce modèle prend en compte le caractère gaussien des faisceaux, l'absorption des guides, et la géométrie variable du couplage. La mesure de l'épaisseur de la couche d'air prise entre le prisme et le guide est primordiale pour l'évaluation de l'efficacité de couplage. Cette mesure a souvent été considérée comme un élément limitant sérieusement l'efficacité de la technique du coupleur à prisme, lorsque celleci nécessite de connaître la puissance guidée dans la couche mince. Ce problème est maintenant résolu, puisque nous avons défini une méthode qui nous permet de déterminer cette épaisseur d'air avec une précision excellente, et en temps réel [3].

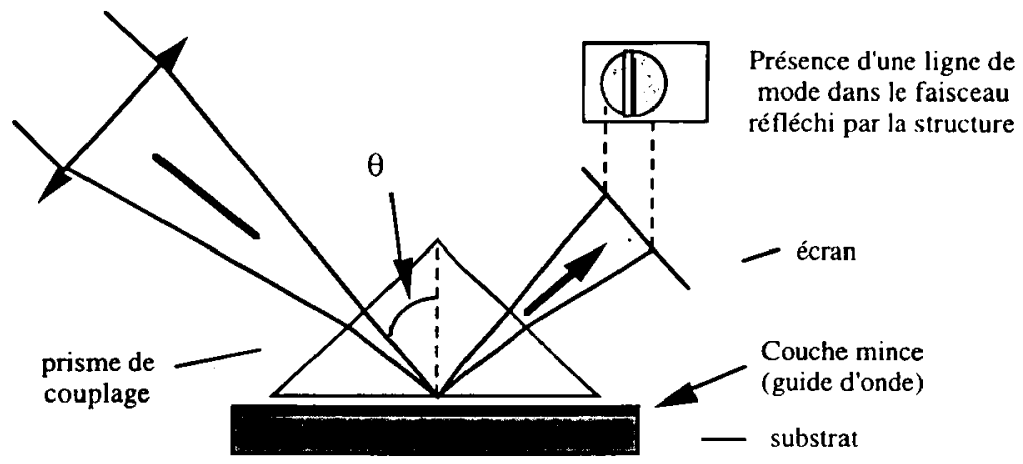

Figure 1: Le coupleur à prisme à réflexion totale permet de coupler de façon résonnante de la lumière dans un guide d'onde optique plan. On peut alors observer une ligne de mode dans le faisceau réfléchi, constituée d'une ligne noire et d'une ligne brillante juxtaposées.

Dans l'hypothèse d'un effet de type Kerr optique, on détermine alors les coefficients d'indice nonlinéaires $\mathrm{n}_{2}$ de matériaux diélectriques, tels que $\mathrm{TiO}_{2}, \mathrm{SiO}_{2}, \mathrm{Ta}_{2} \mathrm{O}_{5}, \mathrm{ZnS}$. Avec nos conditions d'éclairement et compte tenu des temps de relaxation qui sont de quelques millisecondes, on a affaire à des effets d'origine thermique.

Outre cet effet de type Kerr optique, nous observons des phénomènes capables de suivre les modulations du faisceau pompe, qui semblent être dus à des problèmes de désorption d'eau et/ou de dilatation des matériaux. Ces effets, considérés comme lents, ne sont généralement pris en compte que dans le cas d'illuminations par des faisceaux intenses et continus. Etant donnée la haute sensibilité de notre appareillage, ils sont ici parfaitement détectables.

\section{Références}

[1] Rigneault H., Flory F., Monneret S., Appl. opt., 34 (1995), 4358-4368

[2] Flory F., Rigneault H., Massaneda J., Monneret S., Rev. of Las. Eng. 24 (1996), 94-102

[3] Monneret S., Tisserand S., Flory F., Rigneault H., Appl. opt., 35 (1996), 5013-5020 